\title{
Rapid Estimate of Wind Turbine Energy Loss due to Blade Leading Edge Delamination Using Artificial Neural Networks
}

\author{
M. Sergio Campobasso* \\ University of Lancaster \\ Department of Engineering \\ Edmondo Minisci \\ University of Strathclyde \\ Department of Mechanical and \\ Aerospace Engineering \\ Glasgow G1 1XQ, United Kingdom
}

Anna Cavazzini ${ }^{\dagger}$

University of Lancaster

Department of Engineering

Lancaster LA1 4YR, United Kingdom ～Lancaster LA1 4YR, United Kingdom

\begin{abstract}
Estimating reliably and rapidly the losses of wind turbine annual energy production due to blade surface damage is essential for optimizing maintenance planning and, in the case of leading edge erosion, assessing the need for protective coatings. These requirements prompted the development of the prototype system presented herein, using machine learning, wind turbine engineering codes and computational fluid dynamics to estimate annual energy production losses due to blade leading edge delamination. The power curve of a turbine with nominal and damaged blade surfaces is determined, respectively, with the open-source FAST and AeroDyn codes of the National Renewable Energy Laboratory, both using the blade element momentum theory for turbine aerodynamics. The loss prediction system is designed to map a given three-dimensional geometry of a damaged blade onto a damaged airfoil database, which, in this study, features 6000+ airfoil geometries, each analyzed with NavierStokes computational fluid dynamics over the working range of angles of attack. To avoid lengthy aerodynamic analyses to assess losses due to damages monitored during turbine operation, the airfoil force data of a damaged turbine required by AeroDyn are rapidly obtained using a machine learning method trained using the pre-existing
\end{abstract}

*Email: m.s.campobasso@lancaster.ac.uk. Corresponding author.

†Email: a.cavazzini@lancaster.ac.uk

‡Email: edmondo.minisci@strath.ac.uk 
airfoil database. Presented results demonstrate that realistic estimates of the annual energy production loss of a utility-scale offshore turbine due to leading edge delamination are obtained in just a few seconds using a standard desktop computer. This highlights viability and industrial impact of this new technology for managing wind farm energy losses due to blade erosion.

\section{INTRODUCTION}

Wind turbine blades often operate in harsh environmental conditions, being exposed to precipitation that occurs in a variety of forms. Over time, rain, hail and airborne abrasive particles such as sand, can erode blade surfaces, particularly at the leading edge (LE). Recent experimental evidence indicates that LE erosion reduces significantly the aerodynamic performance of wind turbine blades and, thus, the power plant energy production, by more than $20 \%[1,2]$. Significant energy losses due to geometry alterations of the LE surface region can also occur due to insect, dust [3] and ice accretion [4].

Previous research efforts to assess the impact of wind turbine blade surface alterations on aerodynamic power losses have focused on the detrimental effect of ice accretion [5], dust [3,6] and insect debris [7] accumulation, and LE roughness [8-10]. LE erosion also results in significant reductions of the lift and large increments of the drag forces, and can thus dramatically reduce the blade performance, particularly in the outboard region, where the relative air speeds are high and most energy capture takes place. Despite this, however, the study of the energy losses due to blade erosion has received fairly little attention thus far.

Sareen et al. [2] carried out a comprehensive campaign of wind tunnel measurements to assess the aerodynamic performance loss of the DU 96-W-180 wind turbine airfoil subject to geometry alterations due to different patterns (pits, gouges and delamination) and severity (moderate, medium and advanced) of LE erosion. Their eroded airfoil design relied on photographic records of wind turbine blades in operation and eroded blades undergoing repair provided by $3 \mathrm{M}$. These photographic data sets collected from wind power plant operators covered a range of rotor blade sizes up to and including megawatt-scale rotors that had been in operation for 1 to 10+ years. Making use of their airfoil force data based on wind tunnel testing, and the PROPID wind turbine analysis and design code, the authors found that the loss of Annual Energy Production (AEP) of a 2.5 MW class turbine due to LE erosion can approach $25 \%$ of the nominal value. Schramm et al. [11] used OpenFOAM [12], an open-source Navier-Stokes computational fluid dynamics (CFD) package, and the National Renewable Energy Laboratory (NREL) wind turbine engineering code FAST [13] to assess the impact of a particular LE delamination pattern on the AEP of the NREL 5 MW reference turbine [14]. With reference to wind frequency data measured with a met mast at Risø [15], they reported an AEP reduction of about $8 \%$ for the LE damage they considered. Wang et al. [16] developed a pitting erosion geometric model for the CFD analysis of the aerodynamic performance of wind turbine airfoils suffering from this type of surface damage. Each erosion pit was modeled as a semi-circular cavity. Making use of two-dimensional (2D) flow simulations performed with the ANSYS FLUENT incompressible Reynolds-averaged Navier-Stokes (RANS) solver coupled to Menter's $k-\omega$ Shear Stress Transport (SST) turbulence model [17], the authors carried out comprehensive parametric analyses of the dependence of the lift reduction and drag increment of the S809 wind turbine airfoil [18] on the depth, surface density, surface extent, and location (distance from LE) of the erosion pits. For all these parameters, critical values were 
determined above which the airfoil performance did not undergo any further significant reduction.

The studies above highlight that the interest in understanding and quantifying the dependence of wind turbine energy production on blade damage, particularly in the frequent case of damage due to LE erosion, is increasing. However, to the best of the authors' knowledge, there is presently no research into developing a reliable and rapid system for assessing such losses or tackling the challenges of developing this type of system for industrial application. The work presented in this paper aims at addressing this shortfall by presenting and demonstrating the modular wind turbine AEP loss prediction system (ALPS) framework, a novel data- , CFD- and engineering code-based technology for assessing wind turbine energy losses and load variations due to blade surface damage. The information made available by this technology can be used to $a$ ) quantify the revenue reductions due this type of AEP losses and help asset managers deciding when it is most convenient to undertake maintenance, and $b$ ) assess the convenience of adopting safeguards such as LE protections $[11,19]$. The rationale behind the development of ALPS is to provide a tool capable of rapidly and reliably assessing AEP losses due to real blade surface damages with a definition available in the form of three-dimensional (3D) images. Although ALPS has been designed to deal with fairly general surface damage pattern and severity, the demonstration provided in the study below focuses on the AEP losses of a multi-megawatt offshore wind turbine caused by moderate to advanced erosion-induced LE delamination.

The paper is structured as follows: the definition of the architecture, input and output data of ALPS, and the interdependencies of its modules are provided first; this is followed by the specification of the wind turbine computational aerodynamics subsystem, made up of the ANSYS FLUENT [20] incompressible RANS solver and the NREL wind turbine aerodynamics code AeroDyn [21]. The paper continues with the definition of the power control strategy of the turbine with nominal blade geometry. Then, the parametrization of the considered LE delamination damage, and the CFD set-up used for the generation of the force data of 6000+ damaged airfoils making up the initial ALPS database are presented. The machine learning (ML) approach used to extract from the ALPS airfoil repository the force data of the blade cross sections associated with user-given 3D damaged blade geometries is reported next. The Results section demonstrates the applicability of ALPS for real installations by using this novel technology to reliably estimate in a few seconds the power, loads and regulation curves of an offshore wind turbine whose blades feature a general LE delamination damage, representative of those observed in operation. In the same section, the formulation of a possible adaptive power control strategy of wind turbines with blade surface damage, aiming to mitigate the detrimental effect of this damage on AEP, is also presented and demonstrated. The concluding section summarizes this work and provides an overview of future extensions.

\section{ENERGY LOSS PREDICTION SYSTEM}

ALPS is a modular analysis framework consisting of several interlinked modules. Its main function is to determine the annual energy production of a wind turbine whose blades feature surface damage of general shape and severity. The modules of ALPS are indicated in the block diagram of Fig. 1, which shows with arrows module dependencies, and red boxes module outputs. The calculation of the wind turbine power and loads for the entire range of operating wind speeds relies on blade element momentum theory (BEMT) engineering codes (module labeled 'Wind Turbine Model'), which require the lift and drag curves of the airfoils making up the cross sections of the considered damaged blade. The airfoil 
geometry and force database of ALPS (box labeled 'Airfoil Database') contains geometry and force data of airfoils whose shape conforms to the nominal design intent or differs from that because of a blade surface damage developed and/or accrued during turbine operation. The airfoil data presently included in the ALPS database have been computed with RANS CFD, but hybridization methods enabling the concurrent use of experimental and CFD airfoil force data will be investigated in forthcoming extensions.

The main input of ALPS is the current 3D geometry of the blades, consisting of the nominal geometry of the blade and any surface damage (box with enlarged view of delaminated blade LE), with the latter information typically available in the form of blade photographs. These data are passed to a geometry analysis system (GAS), which outputs sectional damage data (e.g. LE delamination extent and depth) for a user-given number of blade cross sections. The GAS module is built upon image processing and CAD software, and it is presently under development. In the ALPS demonstration considered below, the starting point is a set of blade airfoil LE delamination parameters selected on the basis of information available in the literature.

In general, none of the damaged airfoil profiles of an operational turbine will be contained in the current database. Therefore, ALPS uses a machine learning-based method to determine the lift and drag force data of each damaged airfoil making use of the knowledge repository of the airfoil database. In this study, Artificial Neural Networks (ANNs) are used to estimate the values of lift and drag for the whole operating range of the local angle of attack (AoA). The GAS module will include parametric representations of typical blade surface damage patterns, and, for each damaged cross section extracted from the monitored 3D blade geometry, will return the value of the parameters defining the damage of the associated airfoil. This set of parameters, along with a set of operation parameters of the blade sections (e.g. AoA range) form the input of the ANNs module, which returns force data over the requested range of operating conditions.

The force data of the damaged blade sections are then used by the wind turbine module to determine the power curve and the aerodynamic loads of the turbine being assessed. The alterations of the airfoil force data due to blade surface damage like erosion will also result in a variation of the optimal tip-speed ratio and the value of the blade pitch required to limit the power to its rated value above the rated wind speed. It is unclear whether wind turbine power control strategies can presently adapt all of their settings to account for these time-dependent (varying over several months) alterations, but it is expected they will, if they do not do so already. Therefore, in the study below the damaged turbine power control strategy yielding the optimal values of rotor speed below rated wind speed, and the value of the blade pitch limiting the power to its rated value above rated wind speed have been modified in order not to overestimate the AEP loss due to suboptimal turbine control. The modified or adaptive power control algorithm is set by the module labeled 'Control' in Fig. 1, and this modified control strategy is discussed later in the paper. The damaged turbine AEP is then computed by integrating the power curve of the damaged turbine against the wind frequency distribution at the turbine installation site.

\section{WIND TURBINE COMPUTATIONAL AERODYNAMICS}

For each operating condition, namely set values of wind speed, rotor speed and blade pitch, the aerodynamic power and rotor loads are determined with a BEMT code using as input also the lift and drag force data of the nominal or damaged 


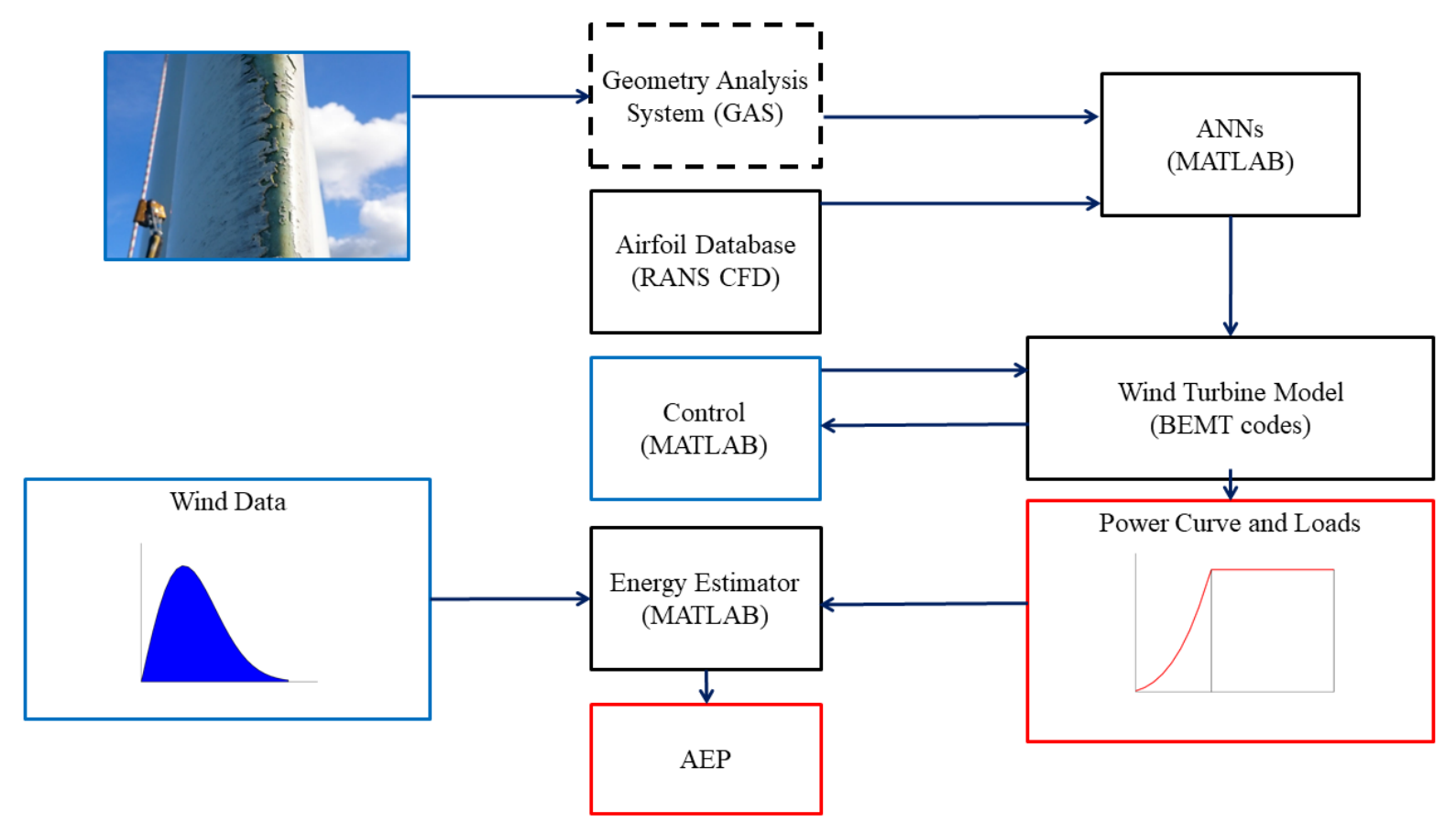

Fig. 1. Definition of ALPS modules and their dependencies.

airfoils corresponding to the rotor blade sections. The force data of the airfoils making up the blade under analysis are determined by means of ANNs, the construction of which, in turn, requires the force data of all airfoils in the ALPS database. The airfoil force data of the ALPS database are determined with Navier-Stokes CFD. The main features of the BEMT and CFD codes used in the analyses reported below are summarized in the following subsections.

\subsection{Navier-Stokes computational fluid dynamics analysis}

Version 19.2 of the finite volume CFD code ANSYS FLUENT [20] is used to determine the flow field and the forces of all considered nominal and damaged airfoils. To obtain these data, the incompressible RANS equations in pressure-based form are solved in conjunction with the $k-\omega$ SST eddy viscosity model for the turbulent closure using unstructured grids. The second order upwind scheme is used for the space-discretization of both the mean flow equations and the turbulence model equations. The adopted numerical integration is based on the coupled algorithm, whereby the momentum and the pressure-based continuity equations are solved in a fully-coupled fashion. The SST model equations are instead integrated in a segregated or loosely coupled fashion.

\subsection{Blade element momentum theory analysis}

The aerodynamic power and loads of turbines with nominal and damaged blade geometry for given value of wind speed, rotor angular speed and blade pitch are determined with AeroDyn [21], the NREL time-domain wind turbine aerodynamics module. Aerodynamic calculations within AeroDyn are based on the principles of actuator lines, whereby the 3D flow field around a slender body is approximated with a local 2D flow model past cross sections, and the distributed pressure and viscous stresses are approximated by lift forces, drag forces, and pitching moments lumped at the node associated with 
the considered 2D cross section. Using this approach, the code can compute aerodynamic loads on both rotor blades and turbine tower. Analysis nodes are distributed along the length of each blade and the tower, the 2D forces and moment at each node are computed as distributed loads per unit length, and the total 3D aerodynamic loads are found by integrating the 2D distributed loads along the length of the considered blade or the tower.

AeroDyn consists of four submodels: $i$ ) rotor wake induction, ii) blade airfoil aerodynamics, iii) tower influence on blade aerodynamics, and $i v$ ) tower drag, and all four modules are used in the AeroDyn analyses reported in this study. For operating (i.e. not parked or idle) wind turbine rotors, AeroDyn calculates the influence of the rotor wake on blade aerodynamics via axial and circumferential induction factors based on the quasi-steady BEMT, which requires an iterative nonlinear solution process coupling modules $i$ ) and $i i$ ). By quasi-steady, it is meant that the induction reacts instantaneously to loading changes. The calculation of the induction factors and resulting inflow velocities and angles relative to the moving blade are based on the local flow field around each analysis node of the considered blade. The effects of local inflow skew, wind shear, turbulence, tower flow disturbances can also be included in the analysis. Glauert's empirical correction with Buhl's modification replaces the linear momentum balance at high axial induction factors. In AeroDyn, 3D flow features are either neglected or captured through corrections inherent in the model (e.g. Prandtl tip and hub loss corrections or skewed wake corrections) or the input data (e.g. rotational augmentation corrections applied to the airfoil force coefficients). The AeroDyn analyses reported below use steady uniform oncoming wind aligned with the rotor axis, i.e. no wind shear and zero yaw error; the airfoil aerodynamics is assumed steady (e.g. no dynamic stall model is used), and the static pressure perturbation of the downwind tower on the blade aerodynamics is included.

\section{WIND TURBINE CONTROL}

The general strategy of the power control algorithm of utility-scale wind turbines aims at maximizing power by maximizing the power conversion efficiency between cut-in and rated wind speeds, and limiting power to the rated value between rated and cut-out wind speeds. In real applications there exist transitional regions before and between these two main regions. In this report, the general power control strategy developed for the NREL 5 MW reference turbine [14] is adopted. However, some alterations to this power control strategy are adopted for the turbine with damaged blade surfaces to avoid overestimating the AEP of these turbines by using the nominal power control settings, which do not maximize the performance of damaged turbines. These alterations are discussed in the Results section.

The profiles of turbine power $P$, low-speed shaft torque $Q$, angular speed $\Omega$, tip-speed ratio (TSR) $\lambda$ and blade pitch $\beta$ against the wind speed $U$ for a turbine with nominal blade shapes are reported in Fig. 2, in which the subscripts c.i., R and c.o. denote respectively cut-in, rated and cut-out speeds. The turbine starts producing power once the wind speed reaches $U_{c . i .}$. In region 2 , starting shortly after wind speed $U_{c . i .}$, the TSR $\lambda$ is kept constant and equal to the value yielding the maximum power coefficient, thus maximizing the harvested power. Therefore, $\Omega$ increases linearly with $U$, and the aerodynamic torque increases quadratically with both $\Omega$ and $U$ in region 2. Region 2, however, is preceded by the transition region 1.5 , in which the aerodynamic torque varies linearly with $\Omega$, and $\Omega$ increases more slowly with $U$ with respect to what occurs in region 2. Similarly, before the power reaches its rated value, the rotor speed starts increasing more slowly with the wind speed 


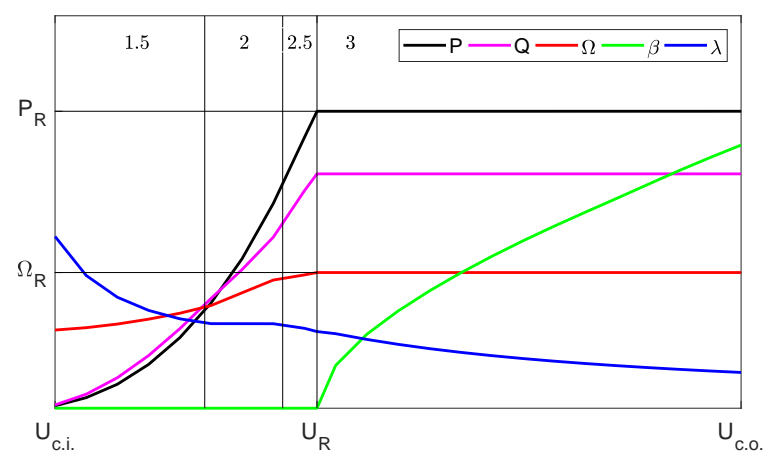

Fig. 2. Wind turbine power control.

(region 2.5) than in region 2. This is because, also in region 2.5, the control algorithm enforces a linear relationship between $Q$ and $\Omega$. Until the rated wind $U_{R}$, the right boundary of region 2.5, is reached, the blade pitch $\beta$ remains constant. As the wind speed increases above $U_{R}$, however, $\Omega$ remains constant, and the blades are actively pitched to feather by the amount required to reduce the lift and its projection on the rotor plane and maintain constant torque and power until the wind speed reaches $U_{\text {c.o. }}$, where the turbine is shut down.

\section{AIRFOIL GEOMETRY AND FORCE DATA}

The subsections below describe the procedure for generating the geometry of a large set of airfoils with delaminated LE making up the ALPS database used in this study, summarize the main numerical settings of the CFD analyses for determining the aerodynamic force coefficients of the damaged airfoils, provide verification and validation analyses of the adopted CFD methodology, and define the ML method used to determine the force data of damaged airfoils whose geometry is not included in the ALPS database.

\subsection{Parametrization of leading edge delamination damage}

The blade surface damage considered herein is LE delamination, a surface alteration appearing more frequently in the advanced stages of LE erosion [2]. The geometry parametrization of LE delamination adopted in this study is sketched in Fig. 3. The damage is defined by 3 parameters, namely its depth $d$, its upper side curvilinear length $s_{u}$, and its lower side curvilinear length $s_{l}$, both measured from the LE.

The baseline airfoils considered in this study are the six airfoils making up the cross sections of the NREL 5 MW reference turbine [14], namely the DU 99-W-405 LM , DU 99-W-350, DU 97-W-300 LM, DU 91-W2-250, DU 93-W-210 LM and NACA64-618 airfoils. The geometry of each of these airfoils is perturbed according to the value range of the delamination parameters reported in Tab. 1. For each delamination stage (mild, moderate and severe), the parameters $s_{u}, s_{l}$ and $d$ are varied independently between the minima and maxima provided in the table with step $\Delta$. All values are normalized by the airfoil chord $c$. Adopting the parameters reported in Tab. 1, 1007 damaged shapes per nominal airfoil geometries are obtained, and the ALPS database has thus 6042 damaged airfoils. Each of these airfoils is generated by a MATLAB code that runs in serial mode on a desktop PC and takes just a few seconds to generate all geometry files.

TURBO-19-1260 


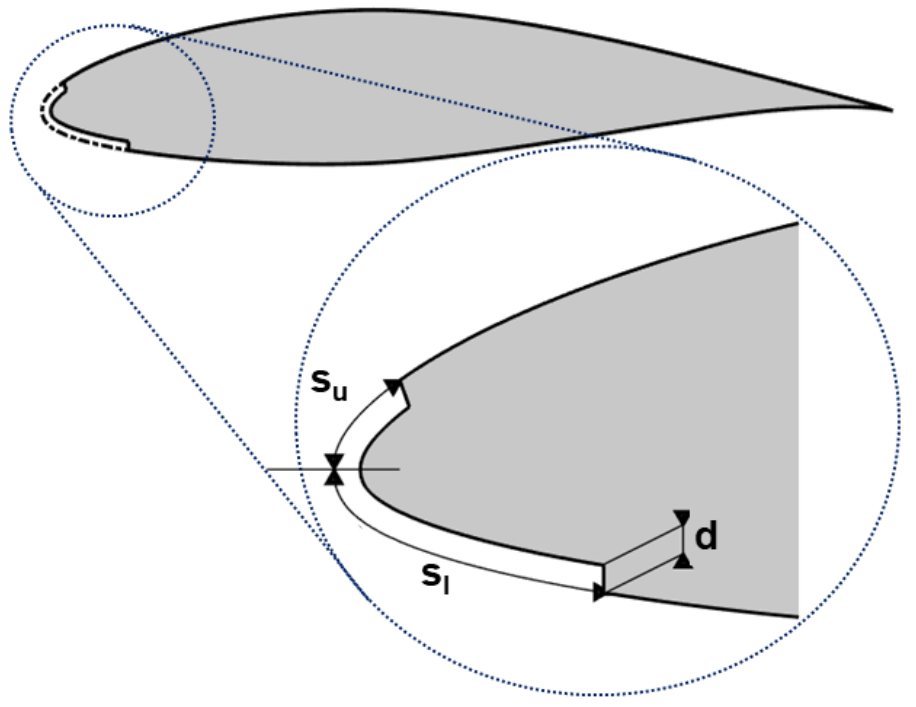

Fig. 3. Geometric parametrization of leading edge delamination.

Table 1. Damage parameter choice for ALPS database.

\begin{tabular}{|c|c|c|c|c|c|c|c|c|c|}
\hline & \multicolumn{3}{|c|}{ Mild } & \multicolumn{3}{c|}{ Moderate } & \multicolumn{3}{c|}{ Severe } \\
\cline { 2 - 10 } & $\min$ & $\max$ & $\Delta$ & $\min$ & $\max$ & $\Delta$ & $\min$ & $\max$ & $\Delta$ \\
\hline$s_{u} / c \times 100$ & 0.5 & 1.0 & 0.1 & 1 & 2.8 & 0.3 & 3 & 6 & 0.3 \\
\hline$s_{l} / c \times 100$ & 0.65 & 1.3 & 0.13 & 1.3 & 3.6 & 0.39 & 3.9 & 7.8 & 0.39 \\
\hline$d / c \times 100$ & 0.1 & 0.1 & - & 0.2 & 0.6 & 0.1 & 0.7 & 1.2 & 0.1 \\
\hline
\end{tabular}

\subsection{CFD set-up}

In this study, the aerodynamic forces acting on each nominal and damaged airfoil are determined with 2D incompressible RANS simulations using ANSYS FLUENT. A Reynolds number of $7 \mathrm{M}$, based on the airfoil chord and the magnitude of the relative velocity and similar to the spanwise averaged value of the NREL $5 \mathrm{MW}$ turbine at rated wind speed, is adopted for all CFD simulations. For each airfoil, a sweep of 16 values of the AoA $\alpha$, from $-10^{\circ}$ to $20^{\circ}$ with a step of $2^{\circ}$ is carried out. Structured C-grids were used in all cases. The C-shaped far field boundary is placed at about 45 chords from the airfoil. A velocity inlet far field boundary condition (BC) is applied on the front, lower and upper parts of the far field boundary, whereas a pressure outlet BC is applied on the rear part. The far field BC data for the SST model consist of a freestream turbulence intensity of $5 \%$ and a turbulent-to-laminar viscosity ratio of 1 . At the airfoil surface, a no-slip condition is enforced. No wall functions are used and the equations of the turbulence model are integrated all the way down to the wall boundary. The adopted wall distance of $3.9 \cdot 10^{-6} c$ of the grid nodes closest to the airfoil surface gives nondimensionalized minimum wall distance $y^{+} \approx 1$ in all cases. All simulations of the ALPS database generated in this study model the flow as fully turbulent, and achieve a reduction of 4 to 5 orders of magnitude of the root-mean-square of both the mean flow equations and the two turbulence model equations. In the majority of the simulations this requires 2000 iterations, while in a few cases, up to 4000 iterations are required.

The grid past nominal airfoils features 151 grid nodes in the normal direction, 327 nodes along the airfoil surface, and 
a total of about $94 \mathrm{~K}$ quadrilateral cells. The grid past airfoils with LE delamination differs from that past nominal airfoils because of an additional structured layer of cells filling the delaminated region. Because of this, these grids feature about 600 nodes along the airfoil and about $126 \mathrm{~K}$ quadrilateral cells. These grid sizes were determined after comprehensive mesh refinement analyses, as summarized below. Views of the grid past the nominal and delaminated NACA64-618 airfoil are reported in Fig. 4-a and Fig. 4-b respectively.

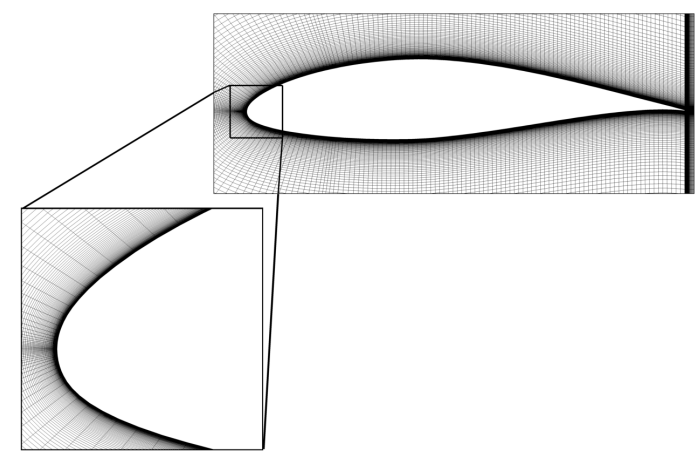

(a) Nominal airfoil geometry.

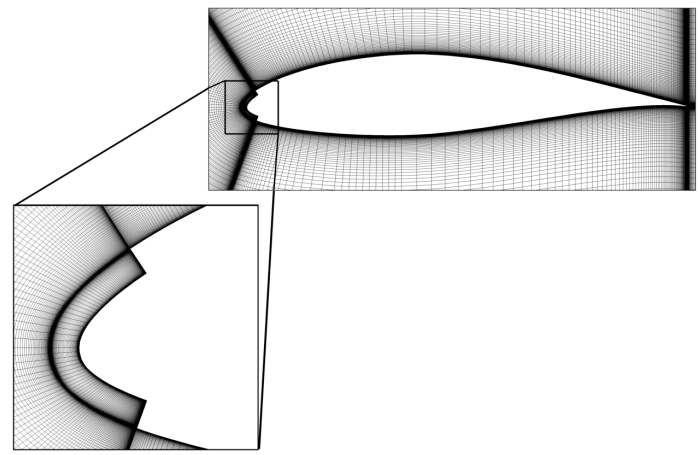

(b) Airfoil with leading edge delamination.

Fig. 4. Enlarged views of computational grids past NACA64-618 airfoil.

In the ALPS airfoil database generated for the demonstration reported in the Results section, a set of 16 AoAs is analyzed for each damaged airfoil, and the database generation thus requires $6042 \times 16=96672$ 2D FLUENT simulations. The process of generating the complete geometry of the physical domain, meshing it, running the CFD simulations is fully automated by means of MATLAB, JAVA, LINUX and ANSYS WorkBench scripting. The computationally most intensive task is the CFD simulation. For this reason, all these simulations are run on the Lancaster University HEC cluster [22]. Using 1 16-core HEC node, one 2D CFD simulation based on the grid settings reported above requires about 4 minutes of wall-clock time, and the construction of the lift and drag curves for each airfoil requires about 64 minutes of wall-clock time. Thus, the generation of the 6042-airfoil force database requires about 6447 hours of wall-clock time using one cluster node. However, the database generation is accomplished by using concurrently 20 cluster nodes, and the required wall-clock time is therefore reduced to about 2 weeks.

\subsection{Verification and validation}

Measured and computed force coefficients of the nominal NACA64-618 airfoil between $\alpha=-10^{\circ}$ and $\alpha=20^{\circ}$ at a Reynolds number of $6 \mathrm{M}$ are reported in the two plots of Fig. 5. The CFD curves labeled 'coarse', 'medium' and 'fine' refer to structured grids featuring $26 \mathrm{~K}, 94 \mathrm{~K}$ and $320 \mathrm{~K}$ quadrilateral cells respectively. The minimum wall distance in the medium refinement grid is $4.4 \cdot 10^{-6} c$, which gives nondimensionalized minimum wall distance $y^{+} \approx 1$ for all AoAs at this Reynolds number. The minimum wall distance in the coarse and fine grids is respectively twice and half the medium grid value. Inspection of these CFD results shows that the medium and fine grid results differ negligibly from each other, confirming that the medium grid settings are adequate for the construction of the ALPS database. With regard to the comparison of 
grid-independent CFD results and experimental data, an excellent agreement of measured and computed $c_{l}$ is obtained over the entire AoA range considered. An overall good agreement of computed and measured drag polars is also observed. All CFD results of Fig. 5 are obtained with the SST turbulence model enhanced by the $\gamma-R e_{\theta}$ transition model [23]. To achieve the good correlation of CFD and experimental data reported below, the constant $a_{1}$ appearing in the definition of the eddy viscosity had to be reduced from its default value of 0.31 to 0.28 , similarly to the guideline in [4].

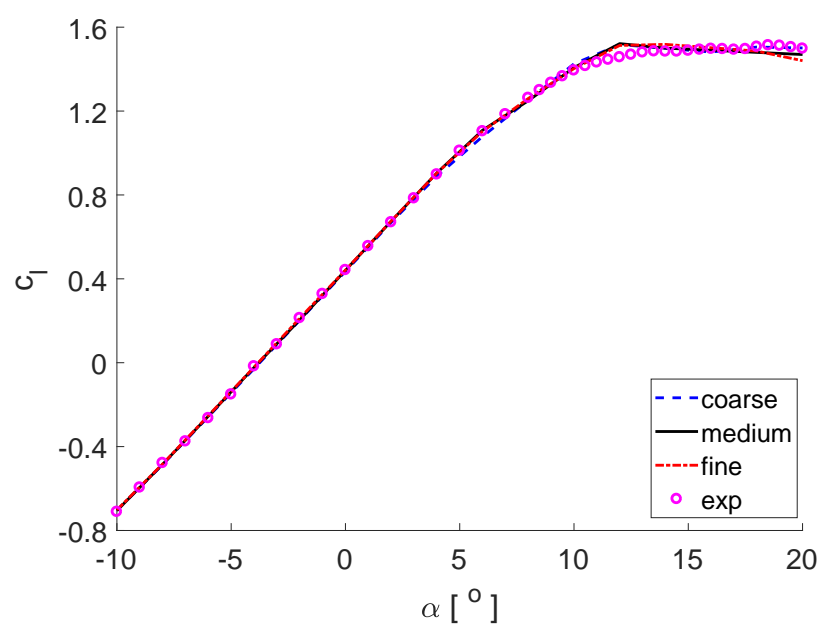

(a) Lift coefficient $c_{l}$ against angle of attack $\alpha$.

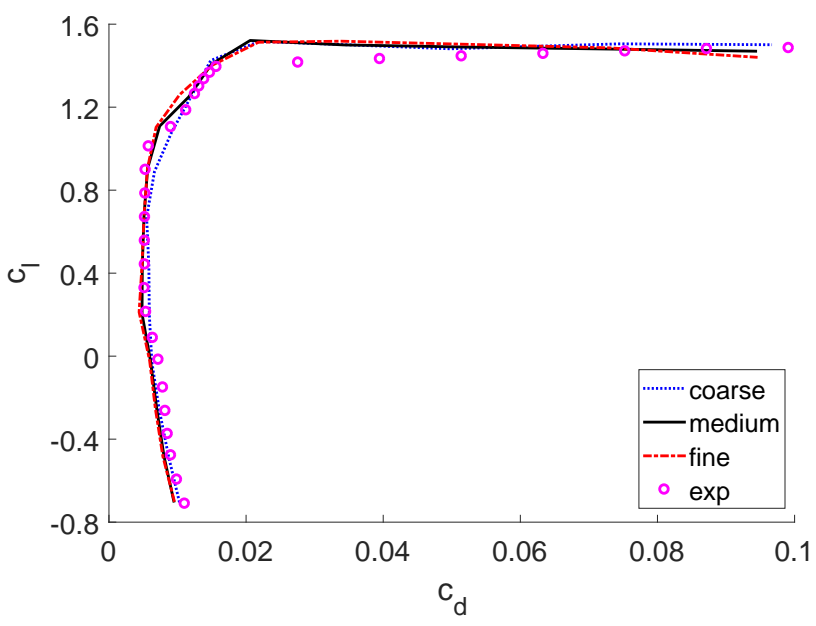

(b) Lift coefficient $c_{l}$ against drag coefficient $c_{d}$ (drag polar).

Fig. 5. Comparison of computed and measured force coefficients of NACA64-618 airoil.

To further verify and validate the ALPS CFD set-up, the analysis of the aerodynamic forces acting on the DU96-W180 airfoil and a damaged variant of this profile at a Reynolds number of $1.5 \mathrm{M}$ is considered next. The damaged variant is affected by LE delamination with damage parameters $s_{u} / c=0.030, s_{l} / c=0.039$ and $d / c=0.00834$, and is an approximation to one of the damaged variants of the DU96-W180 airfoil that were tested in a wind tunned [2]. Computed and measured force coefficients of the nominal and damaged airfoils are compared in Fig. 6, in which all measured data are those reported in [2]. Both CFD simulations use the same transtion model set-up of the nominal NACA64-618 airfoil analysis, and a minimum wall distance of $1.6 \cdot 10^{-5} c$, which results in maximum $y^{+}$of order 1 at all grid nodes around both airfoils. The nominal airfoil analyses in Fig. 6 use a C-grid with 94K quadrilateral cells, and those of the damaged airfoil use a modified C-grid with $126 \mathrm{~K}$ elements. Both results are grid-independent. This was verified by carrying out the nominal airfoil simulations also with a coarse $26 \mathrm{~K}$-element grid and a fine $320 \mathrm{~K}$-element grid, and the damaged airfoil simulations also with a coarse 30K-element grid and a fine 400K-element grid. The curves in Fig. 6 highlight a fairly good agreement between simulations and experiments, particularly for AoAs up to about $8^{\circ}$. The general trends of CFD and measurements are in good agreement, including the prediction of the small negative AoA below which the lift of the damaged airfoil becomes slightly higher than that of the nominal airfoil. The general outcome of the comparison above is considered quite positive, also in light of some uncertainty on the CFD estimates due to insufficient information on the geometry of the damaged airfoil; moreover, it is noted that this airfoil is used in the outboard sections of turbine blades, where the AoA levels are relatively low, and are in the range in which the comparative analysis of nominal and damaged airfoil aerodynamics provides quite good results. 


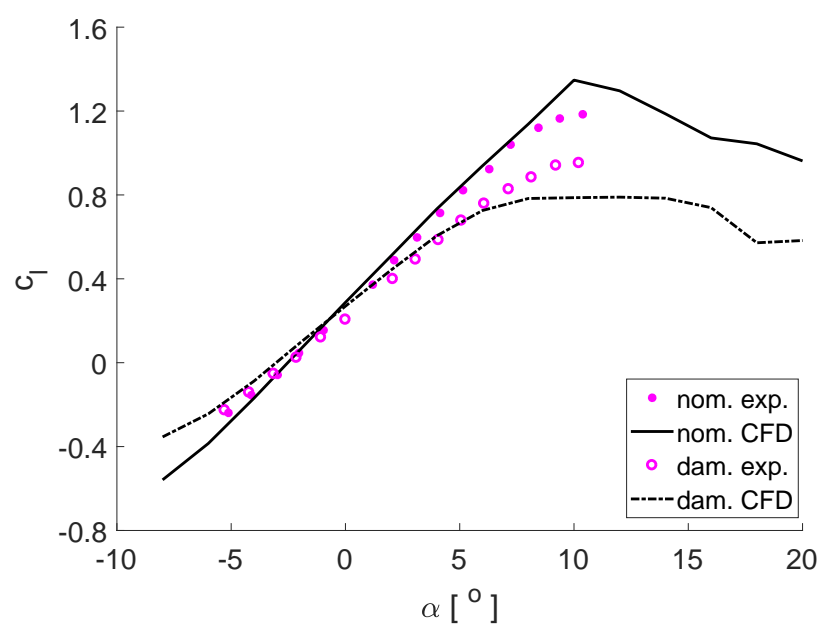

(a) Lift coefficient $c_{l}$ against angle of attack $\alpha$.

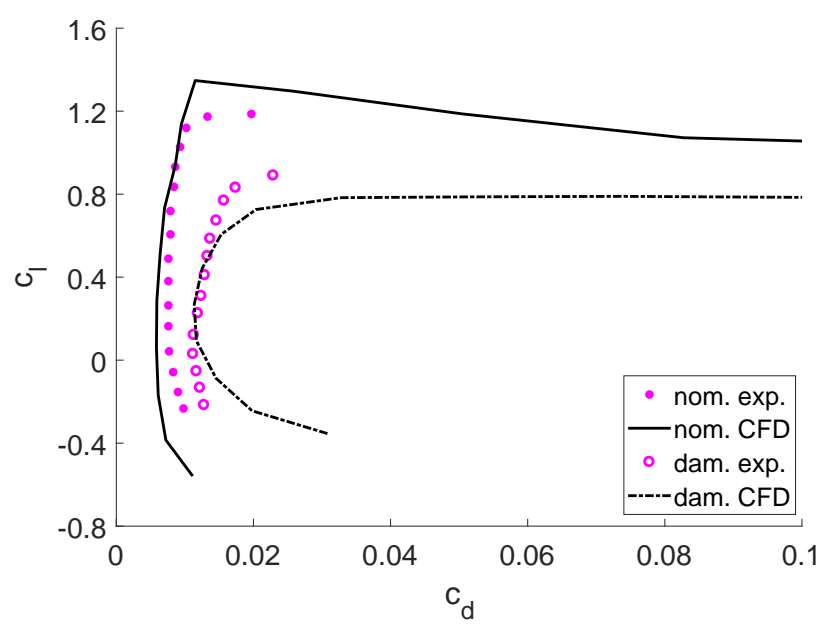

(b) Lift coefficient $c_{l}$ against drag coefficient $c_{d}$ (drag polar).

Fig. 6. Comparison of computed and measured force coefficients of nominal and damaged DU96-W180 airoil.

\subsection{Machine learning approach}

Machine learning uses algorithms and statistical models to develop computer systems that effectively perform a specific task relying on data patterns and inference. Recently, the use of ML in CFD has been rapidly growing, typically with the aim of improving the turbulence modeling prediction capabilities of the computationally affordable RANS technology without incurring the full computational burden of higher-fidelity analyses, such as those based on large-eddy simulation (LES). For instance, ML was used in [24] to achieve more accurate turbulent heat flux film cooling predictions than those of standard RANS linear eddy viscosity models, which assume the heat flux depends linearly on turbulent viscosity and mean temperature gradient, often oversimplifying the physics of the problem. In [25], ML was used to develop nonlinear closures of both turbulence stress and heat flux closures with increased prediction accuracy for trailing edge cooling slot analyses. In both studies, the data sets used to develop the ML models were based on LES data. A recent application of ML to wind turbine engineering was reported in [26], where ML was used in the framework of wind turbine flow control based on actively controlled Gurney flaps. ML was used to build an airfoil force model based on a database of flap-fitted airfoils obtained with CFD and used within a BEMT code for wind turbine analysis.

In this study, ML is used to develop a mathematical model providing rapid estimates of the eroded airfoil force coefficients avoiding time-consuming RANS CFD simulations. Generic multi-layer perception (MLP) feed-forward Artificial Neural Networks with one hidden layer are used in ALPS to learn from the lift coefficient $c_{l}$ and the drag coefficient $c_{d}$ data patterns in the database of damaged airfoils, and infer the aerodynamic forces of eroded airfoils not contained in the database. One $c_{l}$ and one $c_{d}$ ANN model is constructed for each of the six airfoils making up the blade of the NREL 5 MW turbine.

One-layer MLPs like those used herein consist of universal function approximators $\mathbf{f}_{\mathbf{g}}(\mathbf{x}): \mathbb{R}^{n_{d}} \rightarrow \mathbb{R}^{n_{o}}$ [27], where $n_{d}$ is the size of the input vector $\mathbf{x}$, and $n_{o}$ is the size of the output vector function $\mathbf{f}_{\mathbf{g}}$. In the present ALPS version, $\mathbf{x}=\left(s_{u} / c, s_{l} / c, d / c, \alpha\right)^{T}$ and $n_{d}=4$, whereas $n_{o}=1$, since the output function is scalar, corresponding to either $c_{l}$ or $c_{d}$. The 
general matrix-vector definition of $\mathbf{f}_{\mathbf{g}}$ is:

$$
\mathbf{f}_{\mathbf{g}}(\mathbf{x})=A 2\left(\mathbf{b}^{(2)}+\mathbf{W}^{(2)}\left(A 1\left(\mathbf{b}^{(1)}+\mathbf{W}^{(1)} \mathbf{x}\right)\right)\right)
$$

where $\mathbf{W}^{(1)}$ is a weight matrix of size $\left(N \times n_{d}\right)$ and $N$ is the number of neurons in the hidden layer, $\mathbf{b}^{(1)}$ is a bias term represented by a column vector of length $N, \mathbf{W}^{(2)}$ is a weight matrix of size $\left(n_{o} \times N\right), \mathbf{b}^{(2)}$ is a bias term represented by a column vector of length $n_{o}$, and $A 1$ and $A 2$ are the activation functions of the hidden layer and the output layer, respectively. The general schematic of a one-layer MLP feed-forward ANN is depicted in Fig. 7.

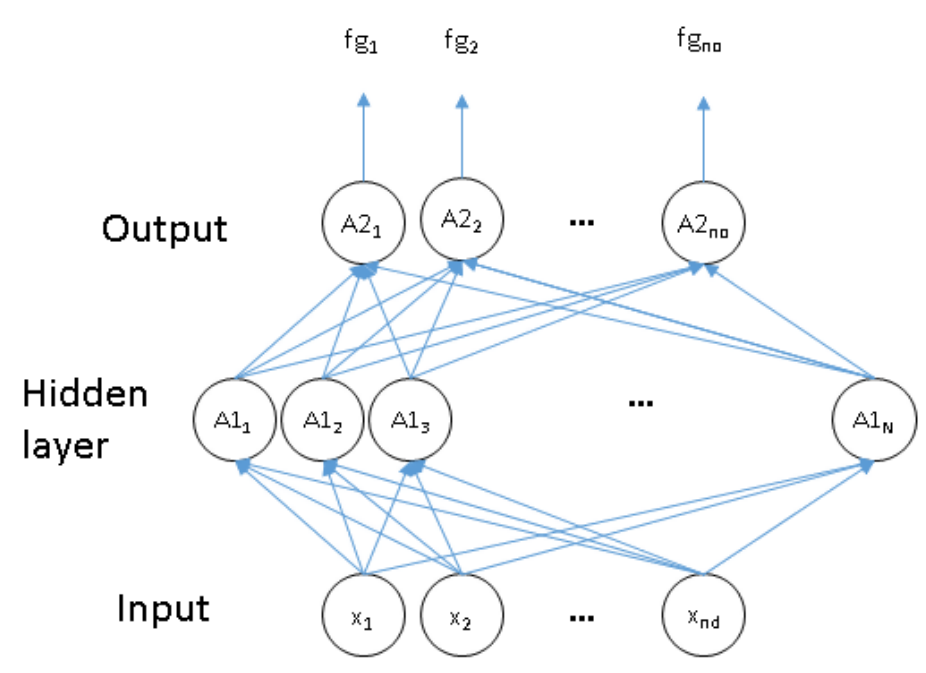

Fig. 7. General schematic of one-layer MLP feed-forward ANNs.

Typically, $A 1$ is the hyperbolic tangent sigmoid function defined as $\tanh (a)=\left(e^{a}-e^{-a}\right) /\left(e^{a}+e^{-a}\right)$, and $A 2$ is linear. With this choice, function $\mathbf{f}_{\mathbf{g}}(\mathbf{x})$ becomes:

$$
\mathbf{f}_{\mathbf{g}}(\mathbf{x})=\mathbf{b}^{(2)}+\mathbf{W}^{(2)}\left(\tanh \left(\mathbf{b}^{(1)}+\mathbf{W}^{(1)} \mathbf{x}\right)\right)
$$

Given a set of $N_{s}$ training samples $\left\{\left(\mathbf{x}_{1}, \mathbf{y}_{1}\right), \ldots,\left(\mathbf{x}_{N_{s}}, \mathbf{y}_{N_{s}}\right)\right\}$, where $\mathbf{y}_{i}$ is the observed response to the input $\mathbf{x}_{i}$, a learning algorithm seeks the values of the weight matrices and bias vectors that minimize the difference between part or all of the observed $N_{s}$ sample responses $\mathbf{y}_{i}$ and the $N_{s}$ responses given by $\mathbf{f}_{\mathbf{g}}\left(\mathbf{x}_{i}\right)$. Gradient-based optimization is often used to determine the weight matrices and the bias vectors by minimizing the aforementioned difference. In this circumstance, since the responses are assumed to be smooth functions of the inputs $\mathbf{x}_{i}$ and the internal weights and biases, the gradients with respect to weights and biases of the difference between sample responses and ML approximations can be computed using the so-called backpropagation method, which relies on applying the chain rule for derivation. The backpropagation approach can 
be applied repeatedly to propagate gradients through all layers starting from the output at the top, where the network gives its response, and working all the way down to the bottom (hidden layer), where the input is provided. Once the gradients with respect to weights and biases for each layer are computed, the objective function expressing the level of fitting of the training data can be optimized.

If the ANN has a sufficiently high number of neurons or degrees of freedom in the hidden layer, a parameter proportional to the chosen number of neurons, the fitting error can be reduced to machine zero; this results in the system overfitting the training data, and being possibly unable to generalize adequately its predictions to regions of the input space where there are no or insufficient training data. To mitigate this risk, two different approaches have been adopted in this study. One approach is to subdivide the available data set into a training set and a validation set. At each step of the gradient-based optimization via backpropagation (training), the fitting error based on the validation set is also computed. The fitting error is taken to be the mean square difference between the observed responses (CFD lift and drag coefficients) and their ML estimates. The system training is then stopped when the fitting error based on the validation set starts increasing. This typically happens before the fitting error based on the training set achieves machine zero. The alternative approach introduces one or more regularization terms that modify the fitting function so that the obtained ANN has good generalization properties. One of the most widely used regularization approaches is Bayesian regularization, in which a modified linear combination of fitting errors, weights and biases is minimized [28].

Using either learning approach to prevent overfitting, the learning process via backpropagation consists of a local gradient-based optimization problem, which is solved with the Levenberg-Marquardt method [29]. Because of the local nature of the method, however, the learning outcome can be affected by the process initialization. To reduce the likelihood of this occurrence, 100 training runs with different random initialization are carried out both with the training and validation set approach, and the Bayesian regularization approach. This is also done with different values of $N$ in both cases to determine the value of this parameter that maximizes the ANN predictive capabilities. The $c_{l}$ and $c_{d}$ ANN models for each of the six sets of damaged airfoils have been selected from those above, and are the two which provide a good trade-off of high fitting of the training set and high generalization properties on the test set. The optimal value of $N$ for both the $c_{l}$ and $c_{d}$ ANN models of the six airfoil sets has been found to vary between 20 and 30. In this study, the size of the training set is $95 \%$ of the randomized database when using Bayesian regularization, and the remaining $5 \%$ forms the test set, used to test the ANN generalization strength. The size of the training set is instead $80 \%$ of the randomized database and that of the validation set is $15 \%$ of the same database when using the approach without regularization, and the remaining $5 \%$ forms the test set.

\section{RESULTS}

To demonstrate the use of ALPS for industrial problems, a moderate to severe LE delamination affecting the blades of the NREL $5 \mathrm{MW}$ reference turbine is considered. The damage is assumed to affect the entire length of the blade, and to have irregular edges (variable $s_{u}$ and $s_{l}$ ) and variable depth $d$. The largest contribution to the rotor power is that of the outboard part of the blades, where erosion is typically stronger. The choice of considering a delaminated LE from hub to tip is made to highlight the modeling flexibility of ALPS. In the demonstration below, the geometry of the delamination damage is taken to 
be the same for all three blades. The case of different delamination patterns on each blade, however, can be easily analyzed using the approach adopted in [30] to analyze the impact on the mean and standard deviation of the turbine power of normally distributed deviations of the blade outer shape from the nominal geometry due to finite manufacturing tolerances. In that study, the performance of a three-blade rotor whose blades are not identical is obtained by taking the arithmetic average of the performance (power and loads) of three different fictitious rotors, each with identical blades affected by a different pattern of blade geometry errors.

The LE damage considered herein has different values of delamination parameters $s_{u} / c, s_{l} / c$ and $d / c$ over 25 radial segments or strips of the blade. The minimum, maximum and mean $(\mu)$ values and the standard deviation $(\sigma)$ of the delamination parameters $s_{u}, s_{l}$ and $d$ of the damage analyzed below are reported in columns 2-4 of Tab. 2, in which all values are normalized by the airfoil chord $c$. A 3D view of the considered delamination pattern is provided in Fig. 8, in which US denotes the view of the blade upper side, and LS that of the blade lower side.

Table 2. Main geometric values of analyzed blade damage.

\begin{tabular}{|c|c|c|c|c|}
\hline & $\min$ & $\max$ & $\mu$ & $\sigma$ \\
\hline$s_{u} / c \times 100$ & 2.71 & 6.4 & 4.44 & 1.02 \\
\hline$s_{l} / c \times 100$ & 2.76 & 7.86 & 5.63 & 1.24 \\
\hline$d / c \times 100$ & 0.4 & 0.97 & 0.61 & 0.2 \\
\hline
\end{tabular}

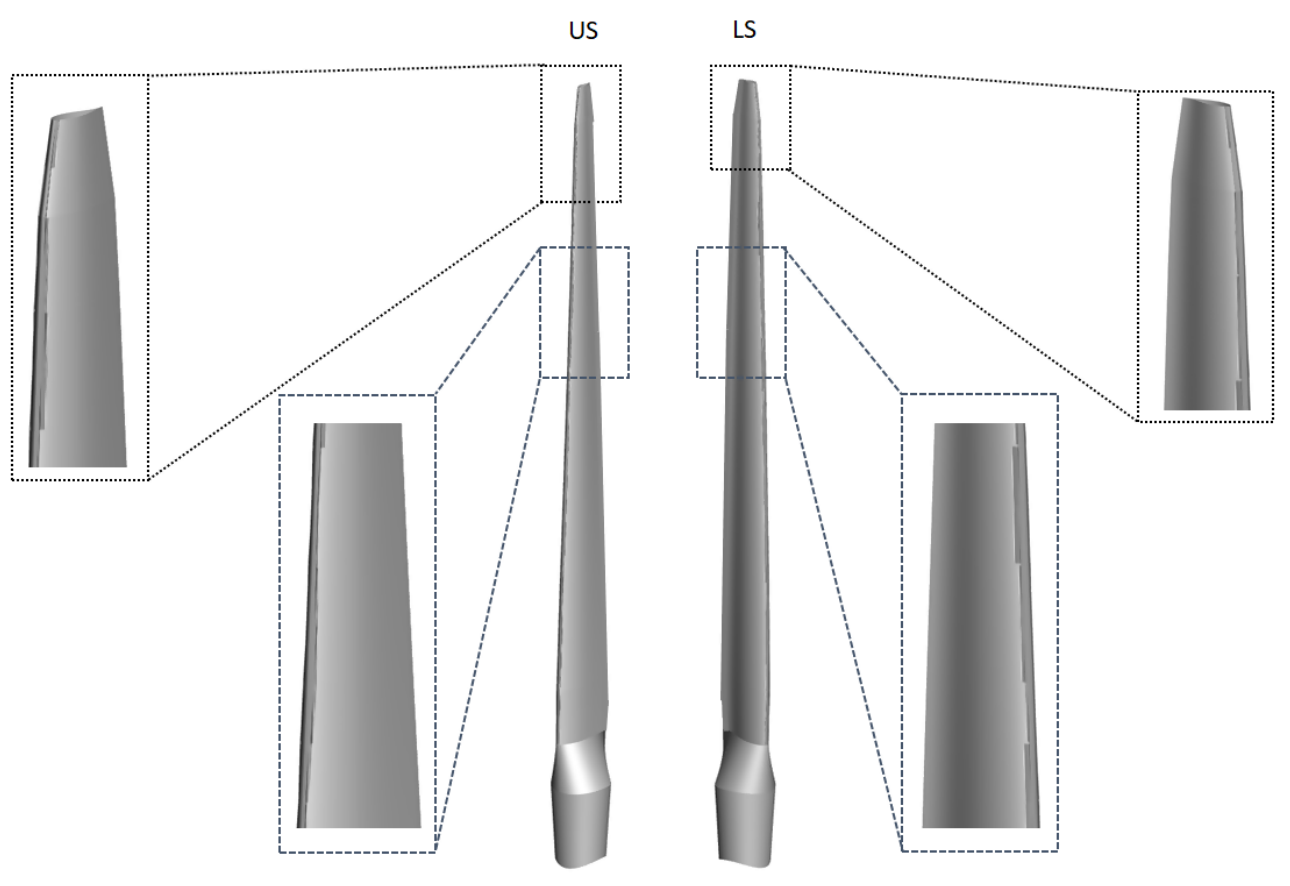

Fig. 8. 3D view of analyzed leading edge erosion damage. 
To assess the reliability of the ANNs based on the ALPS database, the comparison of the lift and drag coefficient curves obtained using CFD and the selected ML approach at six radial positions is considered next. The value of the radius $r$ of the six blade cross sections normalized by the rotor tip radius $R$ is reported in the second row of Tab. 3, and the chord-normalized values of $s_{u}, s_{l}$ and $d$ of each section are reported below the corresponding value of $r / R$. The curves of lift coefficient $c_{l}$ and drag coefficient $c_{d}$ from $-10^{\circ}$ to $10^{\circ}$ of the six sections are depicted in the twelve subplots of Fig. 9. Each subplot provides three curves for the considered force coefficient, namely that of the delaminated section obtained using a CFD analysis (curve labeled CFD-d), that of the delaminated section obtained using ANNs (curve labeled ANN-d), and that of the nominal section obtained using a CFD analysis (curve labeled CFD-n). With regard to the ANNs reliability, an overall excellent agreement between the CFD and ANNs predictions of the force coefficients is observed. With regard to computational cost, it is noted that obtaining the six pairs of $c_{l}$ and $c_{d}$ curves by means of ML, starting from the three delamination parameters of the six damaged airfoils, requires just a few seconds. Conversely, obtaining the same force data using CFD requires about 65 minutes of wall-clock time, resulting from 1 minute to generate 6 grids using concurrently 6 processor cores, and 64 minutes for determining 6 lift and drag curves with 2D CFD simulations using concurrently 6 16-core HEC nodes.

Cross-comparing the force coefficient curves of the damaged and nominal airfoils also shows that for all cross sections $a$ ) the magnitude of the lift coefficient of the eroded airfoils at low and high AoA is lower than that of their nominal counterparts, but is comparable to that of the nominal airfoils for AoA $\alpha$ between about $-5^{\circ}$ and $5^{\circ}$, and $b$ ) a qualitatively similar pattern is observed for the drag coefficients, and the difference between the mean drag coefficient of the damaged and nominal airfoils increases as the blade thickness decreases, i.e. as the blade radius increases.

Table 3. Damage geometry parameters at six radial position
\begin{tabular}{|c|c|c|c|c|c|c|}
\hline & \multicolumn{6}{|c|}{$\mathrm{r} / \mathrm{R}$} \\
\cline { 2 - 7 } & 0.19 & 0.32 & 0.39 & 0.56 & 0.66 & 0.95 \\
\hline$s_{u} / c \times 100$ & 4.9 & 4.3 & 3.8 & 3.7 & 3.0 & 6.4 \\
\hline$s_{l} / c \times 100$ & 5.3 & 4.7 & 4.3 & 4.3 & 6.6 & 6.3 \\
\hline$d / c \times 100$ & 0.4 & 0.4 & 0.4 & 0.52 & 0.61 & 0.85 \\
\hline
\end{tabular}

The curves of the turbine power $P$, torque $Q$ and thrust $T$ against the freestream wind speed $U$ of the damaged and nominal turbines are compared in Fig. 10-a, whereas those of the rotor speed $\Omega$, the blade pitch $\beta$ and the TSR $\lambda$ of the same turbines are compared in Fig. 10-b. An excellent agreement between the power, load and regulation curves of the turbine with blade LE delamination obtained using either CFD or ANNs airfoil data is observed. It is noted that, once the damaged airfoil lift and drag data are determined using the output of relatively lengthy direct CFD simulations or the substantially faster ML approach, the generation of both curve sets requires just a few seconds. For determining the regulation curves of the turbine affected by the given LE damage, AeroDyn requires lift and drag data for 27 airfoils, corresponding to the airfoils at the mean radius of the 25 blade strips, the aerodynamic section at the lowest blade radius and the tip section. The direct CFD method for determining the force data of the 27 airfoils requires a wall-clock time of 129 minutes, resulting from 1 

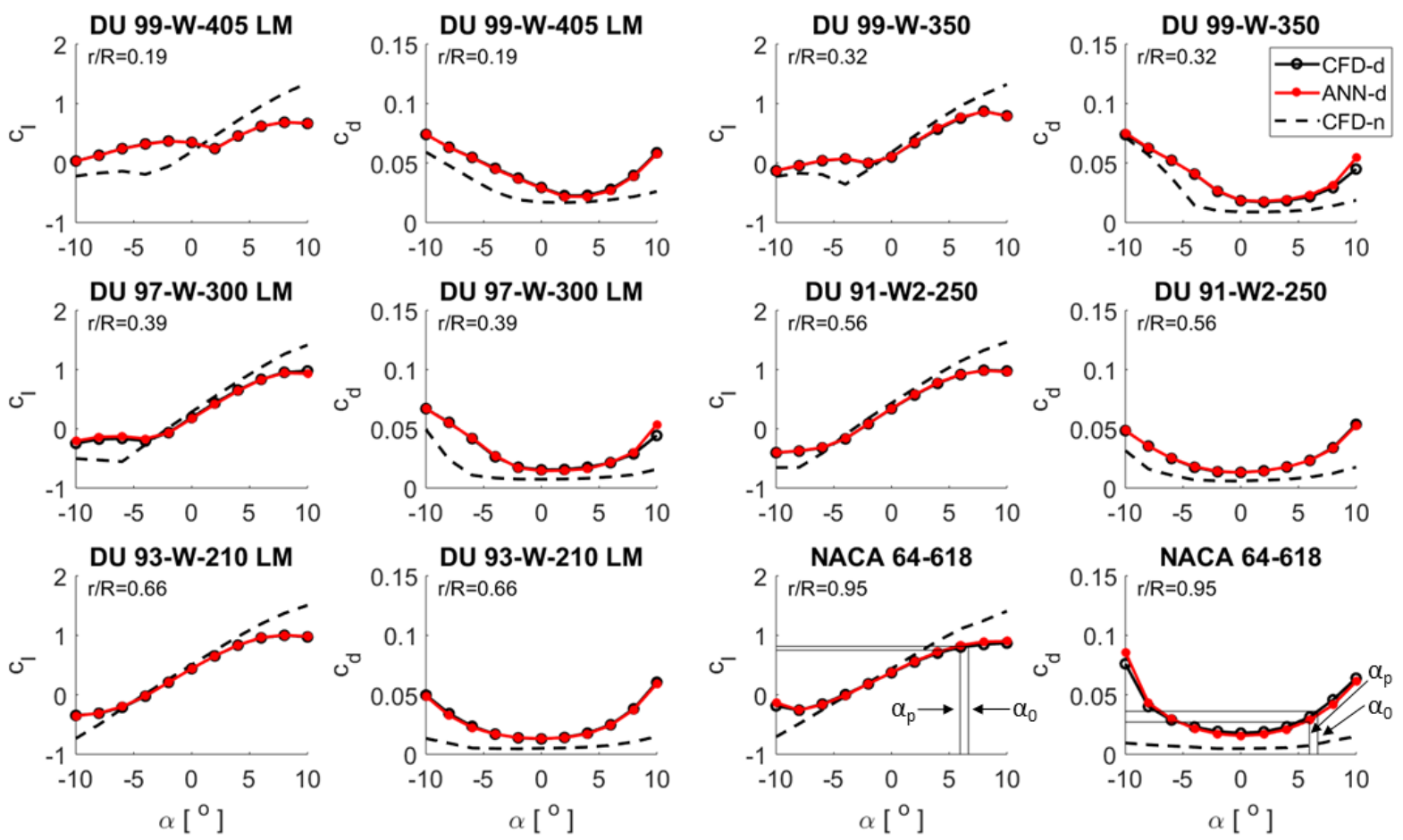

Fig. 9. Comparison of lift and drag forces of nominal and damaged airfoils at six radial positions.

minute for generating the CFD grids and 128 minutes to run 27 CFD analyses, each for 16 values of $\alpha$, using concurrently 20 16-core HEC nodes. Estimating the same force data with ANNs, conversely, requires just a few seconds.

The power control strategy adopted for the turbine with damaged blades is similar to that used for the turbine with nominal blade geometry, but its target settings are adapted to the new rotor aerodynamic characteristics resulting from the sectional force alterations of the blades. To determine the adaptive control settings, the rated aerodynamic power of 5.30 MW, the minimum rotor speed of $6.9 \mathrm{RPM}$, the maximum rotor speed of $12.1 \mathrm{RPM}$ and the cut-off wind speed of $25 \mathrm{~m} / \mathrm{s}$ of the nominal turbine are adopted also for the turbine with eroded LEs. Because of the differences of the lift and drag curves of the nominal and eroded airfoils highlighted above, however, the cut-in and rated wind speeds of the damaged and nominal turbines are slightly different: the cut-in and rated wind speeds of the nominal turbine are 3.0 and $11.4 \mathrm{~m} / \mathrm{s}$ respectively, whereas those of the damaged turbine are $3.2 \mathrm{~m} / \mathrm{s}$ and $13.0 \mathrm{~m} / \mathrm{s}$ respectively. For both turbines, a linear relation between torque and rotor speed is enforced in region 1.5, and such linear relation is maintained until $\Omega$ increases by $30 \%$ over the cut-in value. At the end of region 1.5, $\Omega=9.0$ RPM for both turbines, but $U=7.7 \mathrm{~m} / \mathrm{s}$ for the nominal turbine and $U=7.2 \mathrm{~m} / \mathrm{s}$ for the damaged turbine. For the nominal turbine, the rotor speed in the following region 2 is set to the value corresponding to the known optimal value of $\lambda=7.55$ [14], and, once $\Omega$ achieves $90 \%$ of its maximum value $(\Omega=11.6$ RPM) at $U=10.3$ $\mathrm{m} / \mathrm{s}$, the control uses again a linear relation between torque and rotor speed (region 2.5) until the rated power and maximum rotor speed are achieved at the rated wind speed of $11.4 \mathrm{~m} / \mathrm{s}$. Once the rated power has been achieved, the blade pitch starts increasing to reduce AoAs and maintain the power equal to its rated value as the wind speed increases until the cut-off wind speed. 
For the damaged turbine, the rotor speed in region 2 is set to the value that maximizes the aerodynamic power for each wind speed. This is achieved by wrapping AeroDyn with a MATLAB script that for each wind speed uses the golden section search [31] for gradient-based optimization to determine the optimal value of the rotor speed. This operation is required because the optimal TSR and the corresponding maximum power coefficient of the damaged and nominal turbines are different. The optimal operation parameters of the former turbine are unknown, and they also vary during operation as erosion progresses. Tracking the optimal TSR of the damaged turbine as $U$ increases, one finds that this turbine achieves the maximum rotor speed of $12.1 \mathrm{RPM}$ at $U=10.0 \mathrm{~m} / \mathrm{s}$, well before the rated wind speed of the nominal turbine. Therefore, from $U=10.0 \mathrm{~m} / \mathrm{s}$ to $U=11.4 \mathrm{~m} / \mathrm{s}$, the rotor speed of the damaged turbine is kept constant and equal to its maximum value. At 11.4 $\mathrm{m} / \mathrm{s}$, however, the aerodynamic power of the damaged turbine is still significantly lower than the rated power. To achieve more rapidly the rated aerodynamic power as the wind speed increases above $11.4 \mathrm{~m} / \mathrm{s}$, it is beneficial to start pitching to feather the eroded blades. At a first glance, this seems counterintuitive. The reason for this occurrence is that reducing the AoA at the outer sections of the eroded blade results in the consequent drag reduction outweighing the corresponding lift reduction, and thus in slightly larger torque at lower AoA. This is illustrated in the lift and drag curves of the blade section at $r / R=0.95$ in Fig. 9. The two values of the AoA labeled $\alpha_{p}$ and $\alpha_{0}$ in these subplots are respectively the values computed by AeroDyn using the blade pitch that maximizes power and no pitch at $U=12 \mathrm{~m} / \mathrm{s}$. It is seen that at $\alpha=\alpha_{p}$ a significantly lower drag than at $\alpha=\alpha_{0}$ can be obtained. As mentioned, the lift coefficient at $\alpha=\alpha_{p}$ is lower than at $\alpha=\alpha_{0}$, but due to low twist of the outboard blade sections the reduction of the drag outweighs that of the lift, resulting in an overall higher torque. At $13.0 \mathrm{~m} / \mathrm{s}$, the damaged turbine achieves the rated aerodynamic power. For $U$ varying between 11.4 and $13.0 \mathrm{~m} / \mathrm{s}$, the blade pitch that maximizes the aerodynamic power is obtained by wrapping AeroDyn with a MATLAB script that for each wind speed uses the golden section search to determine the optimal blade pitch. Increasing the wind speed above the rated value of $13.0 \mathrm{~m} / \mathrm{s}$ requires more significant increments of the blade pitch to maintain the power to its rated level. Between the rated and the cut-out wind speed, AeroDyn is wrapped by a MATLAB script that uses Newton's method to determine the blade pitch yielding the rated aerodynamic power.

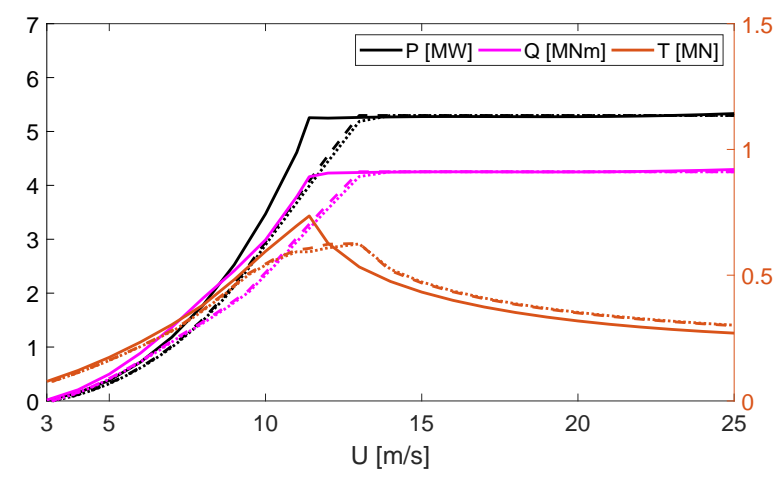

(a) Power $(P)$, torque $(Q)$ and thrust $(T)$ against wind speed $(U)$.

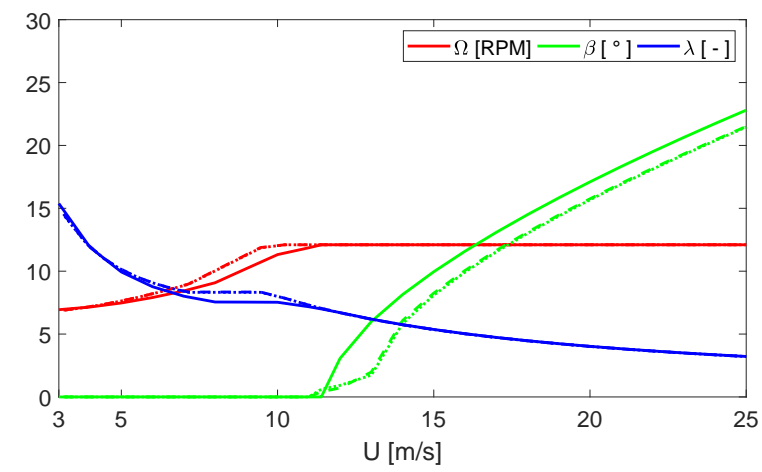

(b) Rotor speed $(\Omega)$, blade pitch $(\beta)$ and TSR $(\lambda)$ against wind speed $(U)$.

Fig. 10. Steady state performance and control curves. Solid, dotted and dashed lines refer respectively to analysis of turbine with nominal blade geometry, damaged blade surface using CFD, and damaged blade surface using ML. 
To explain the reasons for the lift reduction and torque increment accounting for the observed turbine power reduction, the static pressure coefficient $c_{p}$ of the nominal and delaminated blade sections at $r / R=0.8$ for $U=11 \mathrm{~m} / \mathrm{s}$ are compared in Fig. 11, where the origin of the horizontal axis is the nominal airfoil LE. The damaged airfoil has $s_{u} / c=0.027, s_{l} / c=0.078$ and $d / c=0.0076$, and the AoAs of the nominal and damaged sections are $4.9^{\circ}$ and $5.4^{\circ}$, respectively. The suction pressure spike at the front of the delaminated airfoil is caused by the sudden expansion at the forward facing step resulting from the suction side delamination, as shown in the top right corner of Fig. 11. The highlighted recirculation zone after the step reduces the suction side air speed between about 10 and $80 \%$ of the chord with respect to the nominal airfoil case. Thus, the suction side boundary layer of the eroded airfoil is thicker, and between $80 \%$ chord and the trailing edge this results in a flatter pressure profile. All this leads to lower lift and higher drag of the damaged airfoil, with shape drag contributing notably to the overall drag increment.

The AEP loss due to the considered damage is assessed by considering a site with mean wind speed of $9.36 \mathrm{~m} / \mathrm{s}$ over one year, and featuring a Rayleigh wind frequency distribution. These wind data are representative of offshore sites on the north-western coast of England. Integrating the power curve of the nominal and damaged turbines against this wind frequency distribution and ignoring downtimes, gives AEP values of $22191 \mathrm{MWh}$ and $20213 \mathrm{MWh}$. This corresponds to an AEP loss of $8.9 \%$, a value of the same order of magnitude of those reported in the literature. It has also been verified that the AEP loss of the damaged turbine obtained by ignoring the variations of its optimal TSR and power coefficient (i.e. adopting the nominal dependence of the rotational speed on the wind speed from cut-in to cut-out but allowing the pitch controller to limit the aerodynamic power to a constant value of 5.3 MW above rated wind speed) are $9.9 \%$ of the nominal AEP, a value $11 \%$ higher than the loss obtained with the adaptive power control discussed above.

With regard to structural loads, it is noted that the rotor thrust of the nominal turbine reaches a maximum at the rated operating point $(U=11.4 \mathrm{~m} / \mathrm{s})$ before dropping again. This peak is typical of variable generator speed variable blade-pitchto-feather wind turbines because of the transition that occurs in the control system at rated speed between the active generator torque and the active blade pitch control regions. On the other hand, for the considered power control strategy of the damaged turbine, one notes that $a$ ) a fairly flat region of the thrust between the wind speed where the blade pitch starts increasing $(U=11.4 \mathrm{~m} / \mathrm{s})$ and that at which the rated power is achieved $(U=13.0 \mathrm{~m} / \mathrm{s})$ exists, and $b)$ the thrust of the damaged turbine is higher than that of the nominal turbine even before the former reaches rated power, and remains such until the cut-off wind speed.

\section{CONCLUSIONS}

This study has presented ALPS, a novel modular technology for assessing wind turbine energy losses due to blade surface damage. The turbine power curve is determined using BEMT-based engineering codes, such as FAST and AeroDyn. The force coefficients of the damaged airfoils associated with the cross sections of the blades with general LE delamination due to erosion are determined rapidly using artificial neural networks trained using a pre-existing database of damaged airfoil shapes containing airfoil force coefficients for the whole AoA working range. The presented demonstration focused on a radially irregular LE delamination of the blades of the NREL 5 MW turbine, and the analyses revealed that the considered 


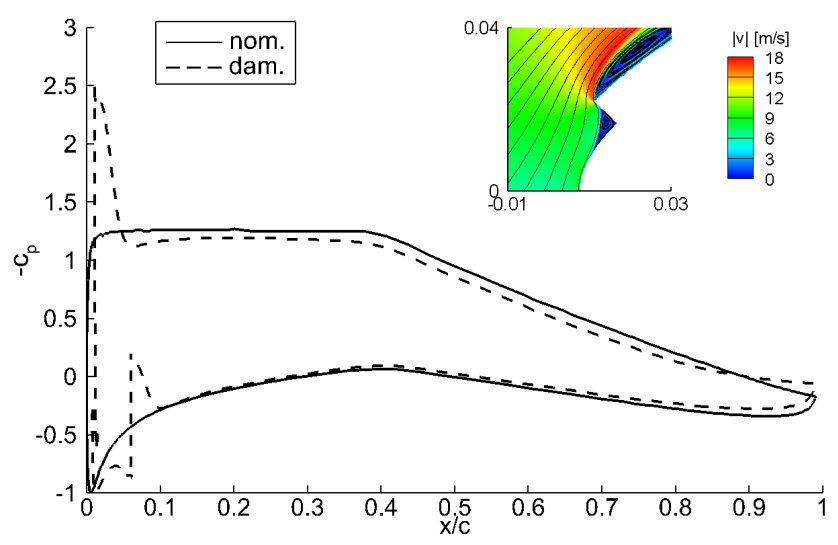

Fig. 11. Static pressure coefficient $c_{p}$ of nominal and delaminated NACA64-618 airfoil at $r / R=0.8$ for $U=11 \mathrm{~m} / \mathrm{s}$, and contours of velocity magnitude $|v|$ in upper leading edge area (top right).

damage of moderate to severe level, reduces the turbine AEP by nearly $9 \%$, a considerable amount which is in line with other measured and computed estimates in the literature. The power control strategy of the damaged turbine was modified between rated and cut-in wind speeds to ensure maximum power also for the damaged turbine. This alteration is needed due to the different optimal TSR and power coefficient of the nominal and damaged turbines. In the case of power controllers lacking this adaptive strategy, thus not accounting for the reduction of the blade aerodynamic efficiency as wear and erosion progress during operation, the actual AEP loss is expected to be higher, by around $11 \%$ of the value recorded using adaptive control. With respect to this, the presented study provides some guidelines on how to make power control more robust to the time-dependent deterioration of the aerodynamic efficiency of the blades.

In the presented demonstration, the ALPS database is generated by means of 2D incompressible RANS flow simulations using ANSYS FLUENT. By automating the geometry and mesh generation phases, the execution of the CFD analyses and the collection of the airfoil force data by means of MATLAB, JAVA, LINUX and ANSYS WorkBench scripting, and by using 20 16-core nodes of the HEC cluster, the 96672 simulations required to determine the lift and drag curves of 6042 damaged airfoils have been carried out in about two weeks of wall-clock time. Once the database has been completed, however, the energy loss of wind farms consisting of hundreds of turbines featuring distinct blade surface damages on each of their rotor blades can be determined in a few minutes at any time of the 25-year lifetime of the wind farm thanks to the extremely low computational cost of the ANN estimates and the BEMT-based engineering analyses. All this results not only is significant case-dependent reductions of the analyses, but also in much higher user-friendliness and portability of the technology, and also reduction of specialist-time due to the automation of the whole process.

A practical issue in the ALPS applicability may arise when the nominal blade geometry is unavailable to the wind farm operator and/or turbine maintenance provider, since this may hinder the generation of the damaged airfoil geometries required to build the airfoil force database. This difficulty may be circumvented by basing the entire damage analysis on a turbine of known geometry, and overall design, control and rated power broadly comparable to the operational turbine under consideration, assuming that the percentage AEP loss is comparable. A second alternative consists of using reverse 
engineering to obtain an approximation to the nominal blade geometry. It is also noted, however, that the trend of wind turbine manufacturers also providing servicing to wind farm operators is rapidly increasing. This occurrence removes the geometry accessibility issue, since these service providers can use ALPS on behalf of wind farm operators to better inform cost analyses and blade material-related choices. In all cases, once the nominal blade geometry is available, the damaged airfoil database generation is a fairly straightforward and inexpensive process, which can be made even more accessible by using open-source software and cloud computing.

The presented study considered only the delamination pattern of the LE damage caused by erosion. In the generation of the ALPS database, the impact of earlier-stage pit and gouge damage patterns can be also analyzed by replacing the 2D RANS CFD simulations with 3D simulations of radially thin constant-chord blade slices featuring different sizes and distributions of these 3D damage patterns. The impact of variable-roughness along damaged blade surfaces can also be accounted for using custom-tailored wall boundary conditions.

Finally, the geometry analysis system module in Fig. 1 was not needed in the presented demonstration, since the ALPS analysis started from the knowledge of the damaged blade sections, rather than from surface definitions of the blade damage. In field applications, the geometry of the damaged blade sections will be provided by GAS, the key link between the damaged blade photographs made available by the condition monitoring provider and ALPS; the GAS implementation will be reported in forthcoming studies.

\section{Acknowledgements}

This work was supported by the Centre for Global Eco-Innovation, funded by the European Regional Development Fund, the UK Engineering and Physical Sciences Research Council in the framework of Lancaster University Impact Acceleration Account, Grant No. EP/R511560/1, and HeliSpeed Ltd, whose initiatives in wind turbine condition monitoring prompted this research programme. Mr. Lorenzo Cappugi is gratefully acknowledged for assisting with the analyses reported in this article. All CFD simulations were performed on the HEC cluster of Lancaster University.

\section{Nomenclature}

AEP Annual Energy Production [MWh].

ALPS AEP Loss Prediction System.

AoA Angle of Attack $\left[{ }^{\circ}\right]$.

ANN Artificial Neural Network.

BEMT Blade Element Momentum Theory.

CFD Computational Fluid Dynamics.

GAS Geometry Analysis System.

LE Leading Edge.

ML Machine Learning. 
MLP Multi-Layer Perception.

$\mathrm{P} \quad$ Turbine power [MW].

Q Torque at low-speed shaft $[\mathrm{MNm}]$.

RANS Reynolds-Averaged Navier-Stokes.

SST Shear Stress Transport.

$\mathrm{T} \quad$ Rotor thrust $[\mathrm{MN}]$.

TSR Tip-Speed Ratio.

\section{References}

[1] 3M, 2011. A 3M Study Is the First to Show the Effects of Erosion on Wind Turbine Efficiency. https://www.pressebox.com/pressrelease/3m-deutschland-gmbh/a-3m-study-is-thefirst-to-show-the-effects-of-erosion-on-wind-turbine-efficiency/boxid/445007. Accessed on 6 May 2019.

[2] Sareen, A., Sapre, C.A., and Selig, M.S., 2014. "Effects of leading edge erosion on wind turbine blade performance". Wind Energy, 17(10), pp. 1531-1542.

[3] Khalfallah, M.G., and Koliub, A.M., 2007. "Effect of dust on the performance of wind turbines". Desalination, 209, pp. 209-220.

[4] Zanon, A., De Gennaro, M., and Kuehnelt, H., 2018. "Wind energy harnessing of the NREL 5 MW reference wind turbine in icing conditions under different operational strategies". Renewable Energy, 115, pp. 760-772.

[5] Jasinski, W.J., Noe, S.C., Selig, M.S., and Bragg, M.B., 1998. "Wind turbine performance under icing conditions". Journal of Solar Energy Engineering,, 120(1), pp. 60-65.

[6] Bak, C., Andersen, P.B., Madsen, H.A., and Gaunaa, M., 2008. Design and Verification of Airfoils Resistant to Surface Contamination and Turbulence Intensity. AIAA paper 2008-7050, August. 26th AIAA Applied Aerodynamics Conference, Honolulu, Hawaii.

[7] Corten, G.P., and Veldcamp, H.F., 2001. "Aerodynamics: Insects can halve wind-turbine powers". Nature Brief Communications, 412, pp. 41-42.

[8] van Rooij, R.P.J.O.M., and Timmer, W.A., 2003. "Roughness Sensitivity Considerations for Thick Rotor Blade Airfoils". Journal of Solar Energy Engineering, 125(4), November.

[9] Somers, D.M., 2004. The S814 and S815 Airfoils. Tech. Rep. NREL/SR-500-36292, NREL, Golden, CO, USA, December.

[10] Fuglsang, P., and Bak, C., 2004. "Development of the Risø wind turbine airfoils”. Wind Energy, 7(2), pp. 145-162.

[11] Schramm, M., Rahimi, H., Stoevesandt, B., and Tamgager, K., 2017. "The Influence of Eroded Blades on Wind Turbine Performance Using Numerical Simulations". Energies, 10(9), pp. 1-15.

[12] OpenCFD Ltd. OpenFOAM - The Open Source Computational Fluid Dynamics (CFD) Toolbox. www . openfoam. org. Accessed on 6 May 2019.

TURBO-19-1260

Campobasso 
[13] Jonkman, J. FAST: An aeroelastic computer-aided engineering (CAE) tool for horizontal axis wind turbines. https : //nwtc.nrel.gov/FAST. Accessed on 6 May 2019.

[14] Jonkman, J., Butterfield, S., Musial, W., and Scott, G., 2009. Definition of a 5-MW Reference Wind Turbine for Offshore System Development. Tech. Rep. NREL/TP-500-38060, NREL, Golden, CO, USA.

[15] Troen, I., and Petersen, E., 1989. European Wind Atlas. Technical Report, ISBN 87-550-1482-8, Risø National Laboratoy, Roskilde, Denmark.

[16] Wang, Y., Hu, R., and Zheng, X., 2017. “Aerodynamic Analysis of an Airfoil with Leading Edge Pitting Erosion”. Journal of Solar Energy Engineering, 139(6).

[17] Menter, F.R., 1994. “Two-Equation Eddy-Viscosity Turbulence-Models for Engineering Applications”. AIAA Journal, 32(8), August, pp. 1598-1605.

[18] Somers, D.M., 1997. Design and Experimental Results for the S809 Airfoil. Tech. Rep. NREL/SR-440-6918, NREL, Golden, CO, USA.

[19] Giguere, P., and Selig, M.S., 1999. “Aerodynamic Effects of Leading-edge Tape on Airfoils at Low Reynolds Numbers". Wind Energy, 2, pp. 125-136.

[20] Ansys-Inc, 2019. Fluent theory guide, release 19.2. https://www. ansys. com/Products/Fluids/ANSYSFluent. Accessed on 6 May 2019.

[21] Heyman, G., Jonkman, B., Murray, R., Damiani, R., and Jonkman, J. AeroDyn: a time-domain wind and MHK turbine aerodynamics module. https: / / nwtc.nrel.gov/AeroDyn. Accessed on 6 May 2019.

[22] Lancaster University. High End Computing (HEC). www. lancaster.ac.uk/iss/hec. Accessed on 6 May 2019.

[23] Menter, F.R., Langtry, R.B., Likki, S.R., Suzen, Y.B., Huang, P.G., and Völker, S., 2006. "A Correlation-Based Transition Model Using Local Variables - Part I: Model Formulation”. Journal of Turbomachinery, 128(3), July, pp. 413-422.

[24] Milani, P.M. and Ling, J. and Saez-Mischlich, G. and Bodart, J. and Eaton, J.K., 2018. “A Machine Learning Approach for Determining the Turbulent Diffusivity in Film Cooling Flows”. Journal of Turbomachinery, 140, February.

[25] Sandberg, R.D. and Tan, R. and Weatheritt, J. and Ooi, A. and Haghiri, A. and Michelassi, V. and Laskowski, G., 2018. “Applying Machine Learning Explicit Algebraic Stress and Scalar Flux Models to a Fundamental Trailing Edge Slot”. Journal of Turbomachinery, 140, October.

[26] Saenz-Auirre, A. and Fernandez-Gamiz, U. and Zuluerta, E. and Ulazia, A. and Martinez-Rico, J., 2019. “Optimal Wind Turbine Operation by Artificial Neural Network-Based Active Gourney Flap Flow Control". Sustainability, 11(10).

[27] Cybenko, G., 1989. “Approximation by superpositions of a sigmoidal function”. Mathematics of Control, Signals, and Systems, 2, pp. 303-314.

[28] MacKay, D., 1992. “Bayesian Interpolation”. Neural Computation, 4, pp. 415-447.

[29] Hagan, M.T., and Menhaj, M., 1994. "Training feed-forward networks with the Marquardt algorithm". IEEE Transac- 
tions on Neural Networks, 5, pp. 989-993.

[30] Campobasso, M.S., Minisci, E., and Caboni, M., 2016. "Aerodynamic design optimization of wind turbine rotors under geometric uncertainty". Wind Energy, 19(1), pp. 51-65.

[31] Giordano, F.R., Fox, W.P., Horton, S.B., and Weir, M.D., 2009. A First Course in Mathematical Modeling. Brooks/Cole, 511 Forest Lodge Rd Pacific Grove, USA. 